\title{
Numerical Simulation of a Self-Similar Cascade of Filament Instabilities in the Surface Quasigeostrophic System
}

\author{
R. K. Scott* and D. G. Dritschel \\ School of Mathematics and Statistics, University of St Andrews, St Andrews KY16 9SS, United Kingdom
}

(Received 12 June 2013; published 11 April 2014)

\begin{abstract}
We provide numerical evidence for the existence of a cascade of filament instabilities in the surface quasigeostrophic system for rotating, stratified flow near a horizontal boundary. The cascade involves geometrically shrinking spatial and temporal scales and implies the singular collapse of the filament width to zero in a finite time. The numerical method is both spatially and temporally adaptive, permitting the accurate simulation of the evolution over an unprecedented range of spatial scales spanning over ten orders of magnitude. It provides the first convincing demonstration of the cascade, in which the large separation of scales between subsequent instabilities has made previous numerical simulation difficult.
\end{abstract}

DOI: 10.1103/PhysRevLett.112.144505

PACS numbers: 47.11.-j, 47.20.Cq, 47.32.C-

The surface quasigeostrophic system is a two-dimensional active scalar equation developed as a model for the slow, large-scale motions of rotating, stratified flows of planetary atmospheres and oceans near a horizontal surface or strong jump in stratification such as the midlatitude tropopause [1-3]. It may be expressed by the material advection of a surface temperature field $\theta(x, y, t)$ by a flow to which it is diagnostically related:

$$
\frac{\partial \theta}{\partial t}+J(\psi, \theta)=0, \quad \theta=-(-\Delta)^{1 / 2} \psi,
$$

where $\psi$ is the stream function, $\Delta$ is the two-dimensional Laplacian, and $J(\cdot, \cdot)$ is the Jacobean determinant [4]. It differs from the usual two-dimensional Euler equations of incompressible fluid motion through the relation between $\theta$ and $\psi$, where the more local relation in the surface quasigeostrophic case gives rise to more energetic small-scale motions. In fact, the advection term in Eq. (1) possesses a quadratic degree of nonlinearity similar to that of the three-dimensional Euler equations, for vorticity in the case of the Euler equations and for the skew gradient of temperature in the case of the surface quasigeostrophic equations [5-7]; see, in particular, Eq. (13) of Ref. [6]. The similarities between Eq. (1) and the three-dimensional Euler equations have stimulated much research into the regularity of the surface quasigeostrophic system, in particular, whether a singularity in the temperature gradient $\nabla \theta$ may form in finite time from smooth initial conditions, or whether weak solutions may possess a finite dissipation anomaly in the inviscid limit, the counterpart to the famous Onsager conjecture for turbulent flow governed by the three-dimensional Euler equations [8,9]. Despite the reduced dimensionality, however, the presence of singularities in the surface quasigeostrophic system remains an open problem.

When the initial distribution of $\theta$ is smooth, it is known that a singularity may not form in finite time if the geometry of the temperature field takes the form of a closing saddle, involving the growth of unbounded gradients of $\theta$ along a line [10]. Numerical studies of the closing saddle [9,11] indicate double exponential growth $\nabla \theta \propto \exp \exp t$, or an even less rapid evolution with time. Singularities may yet form at isolated points. One scenario for this has been proposed involving a cascade of repeated filament instabilities of geometrically shrinking scale $[4,12,13]$. Because filament growth rates scale with the inverse filament width, were such a cascade of instabilities to follow a self-similar pattern, then a singularity involving the blowup of $\nabla \theta$ would occur in finite time. However, a large-scale separation between subsequent instabilities (see below) means that such a cascade has been extremely difficult to demonstrate numerically, the most recent results being limited to at best two or three consecutive instabilities before the limits of numerical resolution are reached [14]. Furthermore, uncertainties remain over the role of the numerical grid in the triggering of each instability.

In the special case where the temperature distribution comprises a discrete patch (uniform inside a closed contour, zero outside, for example), the relation between $\theta$ and $\psi$ may be expressed in terms of a single contour integral around the patch boundary, in a manner directly analogous to the Biot-Savart law relating electric current to magnetic field. Representing the patch boundary by a closed contour $\mathcal{C}$, its evolution is then governed by

$$
\frac{d \mathbf{x}_{i}}{d t}=\frac{\theta_{0}}{2 \pi} \int_{\mathcal{C}} \frac{d \mathbf{x}^{\prime}}{\left|\mathbf{x}_{i}-\mathbf{x}^{\prime}\right|}
$$

for each point $\mathbf{x}_{i}$ on the contour $\mathcal{C}$. Although the discontinuity in $\theta$ at the patch boundary implies a logarithmic divergence of the velocity component tangential to $\mathcal{C}$ [15], this component has no effect on the boundary evolution, which is determined entirely by the component normal to $\mathcal{C}$, and the problem is well posed. The regularity problem for this system is concerned with the formation in finite time 
of a discontinuity in the patch boundary, either through the development of a corner with infinite curvature at a point, or the approach to zero of the minimum distance between two contour segments. In contrast to the smooth case examined in Ref. [14], the filament cross section formed by instabilities of the patch is guaranteed to retain the same trivial profile throughout the evolution. Further, the reduction of the dynamics in the patch case to the evolution of a single contour enables the application of a highly efficient Lagrangian contour dynamical algorithm that is here able to capture the patch evolution through a series of instabilities spanning a range of spatial scales on the order of $10^{10}$. The cascade is shown to be self-similar upon a uniform rescaling of the independent variables.

For completeness, we note that another potential route to singularity for the patch was identified in numerical experiments of Ref. [16], involving the formation of a corner. While mathematically singular, it is important to recognize that this type of singularity has no counterpart in the evolution of the continuous $\theta$ distribution of most relevance to fluid motion: a continuous distribution may develop a corner in a level set of $\theta$ without any accompanying blowup of the temperature gradient. One reason that the instability cascade is important is that the counterpart in the evolution of the continuous distribution may also exist (see below). The extension of the present result to the more general case of the continuous distribution will be addressed in future work.

We solve the evolution of the patch using the contour dynamics method of Dritschel [17], representing the patch boundary by a series of points along a contour that are redistributed according to the boundary shape. The method has been refined here in that the integral [Eq. (2)] around $\mathcal{C}$ is now computed along a series of global splines connecting the points, ensuring continuity of curvature and the tangent vector at each point. The point distribution along $\mathcal{C}$ is determined adaptively according to the curvature of $\mathcal{C}$, the local value of the strain field, and the minimum separation between adjacent segments of the boundary, more points being added to maintain accuracy as the complexity of the boundary increases. This ensures that accuracy is maintained even in regions of very high curvature and where filaments become narrow.

To illustrate the cascade, we consider a patch of $\theta$ defined by an initial elliptical boundary of aspect ratio $a=0.16$, as shown in the upper left panel of Fig. 1, with $\theta=2 \pi$ inside the boundary and $\theta=0$ outside. As time increases, the ellipse rotates and deforms, with the tips developing into two distinct lobes separated by a filament $(t=1.44)$ that subsequently lengthens and thins $(t=1.618$ to $t=1.745)$. By $t=1.774$, an instability of the central filament has occurred with the development of a series of new lobes connected by new filaments at a much smaller scale (inset). These filaments subsequently become unstable and roll up in a self-similar manner on a shrinking time scale (an
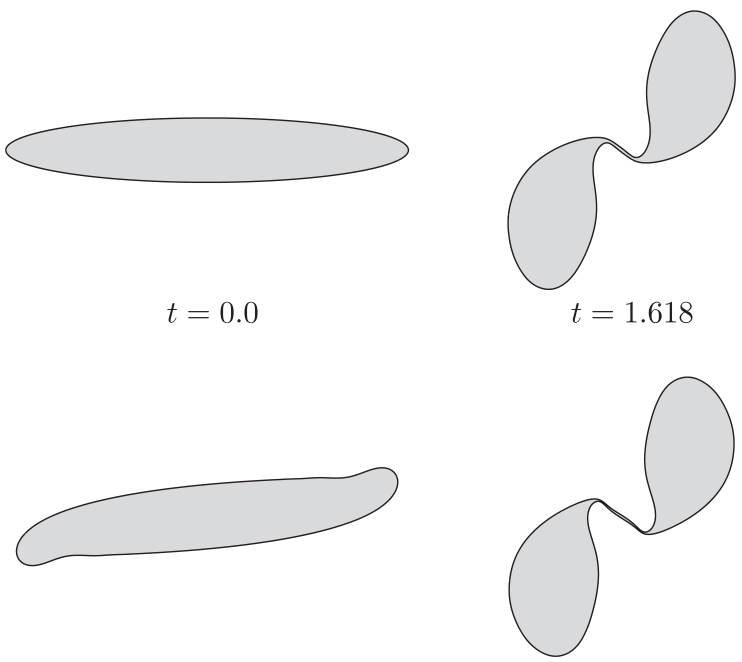

$t=0.23$

$t=1.683$
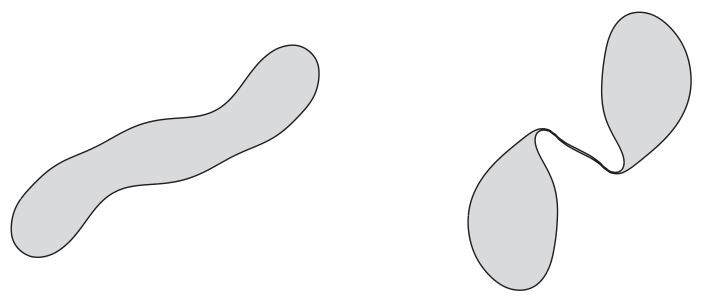

$t=0.87$

$t=1.745$

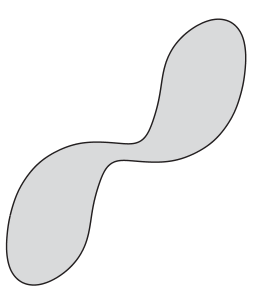

$t=1.44$

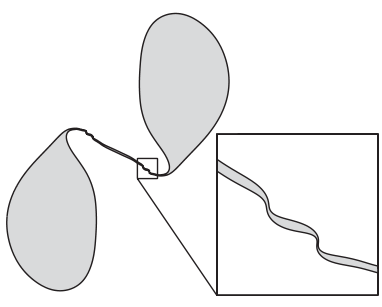

$t=1.774$

FIG. 1. Snapshots of the patch boundary at selected times prior to the onset of the first filament instability; $\theta=2 \pi$ inside the patch boundary and $\theta=0$ outside. The final frame shows an $8 \times$ magnification of a portion of the central filament.

animation of the patch evolution is available in the Supplemental Material [18]).

The rapid collapse of both time and length scales can be seen by considering the growth of curvature $\kappa$ and inverse minimum cross-filament width $d^{-1}$ in time. The development of the filament instability between $t=1.745$ and $t=1.774$ involves the accelerating growth in both $\kappa$ and $d^{-1}$; see Fig. 2(a). At around $t=1.774$, this growth slows as the new filament is more gradually extended. Fast growth begins again at around $t=1.791$ with the onset of the next filament instability; the shrinking time scales suggest unbounded growth in both $\kappa$ and $d^{-1}$ at a finite time $t_{s}$, which is here estimated as $t_{s}=1.79171342353339$; both $\kappa$ and $d^{-1}$ follow approximately $1 /\left(t_{s}-t\right)$, although with alternating periods of more or less rapid growth 
according to whether the smallest filament is in a roll-up or extending phase.

The evolution as the singularity is approached is clarified by introducing a rescaled time variable [16] $\tau=-\log \left(t_{s}-t\right)$. The repeated stages of rapid growth (instability development) followed by a saturation stage (filament extension prior to the onset of the next instability) occur multiple times, with the filament scale shrinking by a factor of around 20 each time; see Fig. 2(b). At each stage, the new filament becomes unstable when it shrinks to a width of about $1 / 20$ of that of the parent filament, independent of the scale of the filament relative to the original ellipse. The time taken for the development of each filament is also $1 / 20$ of that of the previous one, implying a collapse of filament width to zero in a finite time.
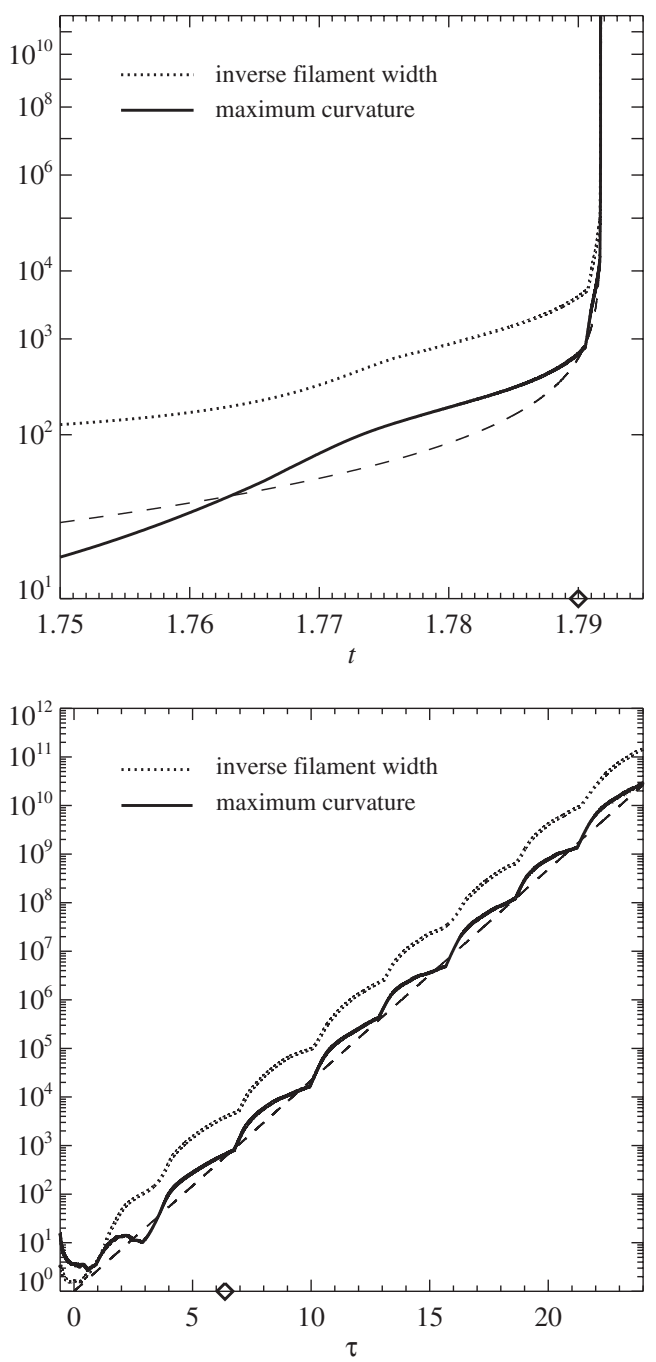

FIG. 2. Maximum curvature and inverse filament width as a function of (a) time $t$ and (b) rescaled time $\tau=-\log \left(t_{s}-t\right.$ ), with $t_{s}=1.7917134235333878$. Corresponding times in each panel are indicated by the diamond symbols; the dashed lines indicate the function $1 /\left(t_{s}-t\right)$. Note the double-log scale in (a) and the single-log scale in (b).
Approaching the singularity time $t_{s}$, the rapid acceleration of the evolution at the smallest scales means that the largescale field becomes effectively frozen. The full evolution up to this time is presented in the Supplemental Material [18], which makes a continuous rescaling of the spatial variables by the instantaneous value of the maximum curvature $\kappa$. Near the final time of the integration $t=1.791713423$ 51654 , or $t_{s}-t=1.7 \times 10^{-11}$, the shape of the boundary is as shown in Fig. 3, where the series of panels depicts successive magnifications by a linear factor of 20 centered on the point of maximum curvature, each central shaded square being magnified in the adjacent panel of the same color. Despite differences in the details of the filament evolution, the large-scale structure at each level of magnification is remarkably uniform. Note that each magnification appears rotated through a constant angle, implying a uniform angular velocity in the rescaled time $\tau$.

The physical cause and uniform scale reduction of the instability cascade may be understood from consideration of the stability properties of a single filament in the surface quasigeostrophic system. Linear growth rates for the filament instability scale as the inverse filament width: the inversion relation in Eq. (1) means that velocity values scale as $\theta_{0}$, which implies that the times taken for disturbances to grow to amplitudes comparable to the filament width are proportional to the filament width itself. We note that such a cascade is typically precluded in the two-dimensional vorticity equation because of the stabilizing effect of background shear on the growth of filament disturbances [19] and because growth rates do not increase as the filament width decreases.

The independence of the rescaling factor on scale suggests that each instability is governed principally by the local shape of the patch in the vicinity of the filament. Because interactions in the quasigeostrophic model are more local than for the case of two-dimensional vortex dynamics, the evolution of a given filament is to a good approximation controlled by the influence of the parent filament that spawned it. However, the contribution to the velocity field by a local approximation to the contour integral, in fact, diverges logarithmically, meaning that the precise evolution requires consideration of the global patch shape. The dynamics is thus expected to be nonuniversal and may depend upon details of the initial conditions. Indeed, for initially elliptical patches, a variety of different evolutions is obtained for different values of the aspect ratio $a$. For large enough $a$, the evolution appears to remain smooth for all time; for $a$ in the range $(0.17,0.19)$, the patch boundary develops a corner before disturbances of the central filament have time to grow to large amplitude; for $a$ below about 0.12 , a more complex combination of corner formation and filament instability occurs. A full description will be reported in a subsequent paper.

While a numerical demonstration can never be a substitute for a rigorous mathematical proof, the present work provides the strongest evidence to date that the instability 


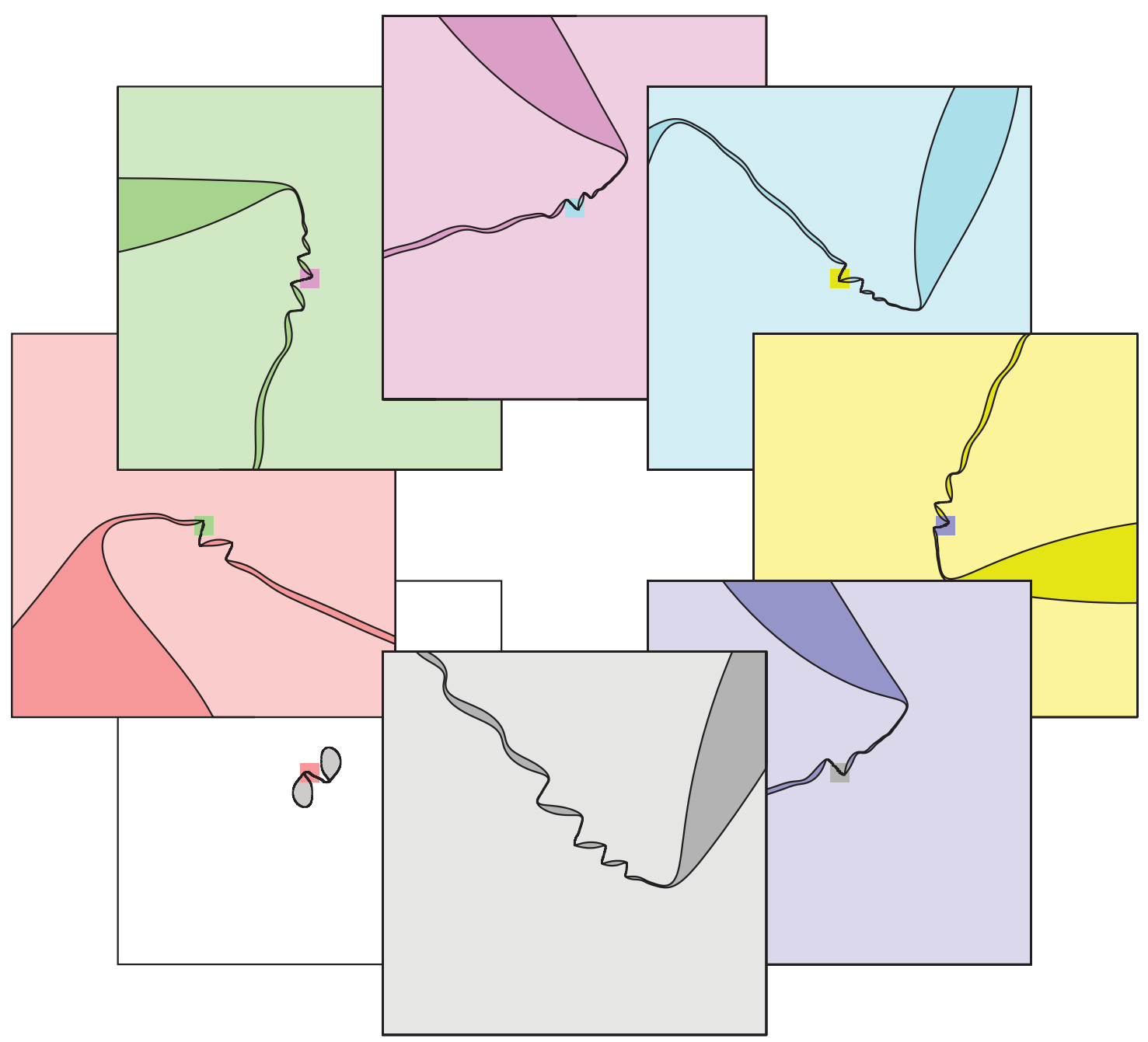

FIG. 3 (color). Contour shape at time $t=1.79171342351654$, or $\tau=24.8068$, at successive magnifications of 20× (linear dimension). The central shaded square in each panel is shown magnified in the adjacent panel of the same color.

cascade may exist in the case of the temperature patch. The existence of such a cascade would, in turn, have implications for the regularity problem of the quasigeostrophic equations when the initial $\theta$ is a smooth distribution in space, a problem about which very little is known. Because $\theta$ is conserved on fluid particles, a collapsing filament with $\theta$ smoothly distributed across its width will retain its initial peak $\theta$ value at the filament center. As pointed out in Ref. [14], the filament boundary, or zero contour of the $\theta$ field, may be identified with the patch contour described above, in which case an instability cascade would again be expected to lead to the unbounded growth of $\nabla \theta$ in finite time. This is in contrast to the case of the corner singularity of Ref. [16], which is intrinsic to the case of the temperature patch and which has no counterpart in the case of an initially smooth $\theta$ distribution. The present results suggest, therefore, that the evolution of the initially smooth $\theta$ distribution may also develop a singularity in finite time, an open problem of considerable theoretical interest. *rks@mcs.st-and.ac.uk

[1] J. G. Charney, Geofysiske publikasjoner 17, 1 (1948).

[2] B. J. Hoskins and F. P. Bretherton, J. Atmos. Sci. 29, 11 (1972).

[3] W. Blumen, J. Atmos. Sci. 35, 774 (1978).

[4] I. M. Held, R. T. Pierrehumbert, S. T. Garner, and K. L. Swanson, J. Fluid Mech. 282, 1 (1995).

[5] P. Constantin, A. J. Majda, and E. Tabak, Nonlinearity 7, 1495 (1994).

[6] P. Constantin, Physica (Amsterdam) 86D, 212 (1995).

[7] C. V. Tran, D. G. Dritschel, and R. K. Scott, Phys. Rev. E 81, 016301 (2010).

[8] L. Onsager, Nuovo Cimento 6, 279 (1949).

[9] K. Ohkitani and M. Yamada, Phys. Fluids 9, 876 (1997).

[10] D. Cordoba, Ann. Math. 148, 1135 (1998).

[11] P. Constantin, Q. Nie, and N. Schorghofer, Phys. Lett. A 241, 168 (1998).

[12] J.-M. Hoyer and R. Sadourny, J. Atmos. Sci. 39, 707 (1982).

[13] R. T. Pierrehumbert, I. M. Held, and K. L. Swanson, Chaos Solitons Fractals 4, 1111 (1994).

[14] R. K. Scott, J. Fluid Mech. 687, 492 (2011). 
[15] M. N. Juckes, J. Atmos. Sci. 52, 3247 (1995).

[16] M. A. Cordoba, D. Fontelos, A. M. Mancho, and J. L. Rodrigo, Proc. Natl. Acad. Sci. U.S.A. 102, 5949 (2005).

[17] D. G. Dritschel, Comput. Phys. Rep. 10, 77 (1989).
[18] See Supplemental Material at http://link.aps.org/ supplemental/10.1103/PhysRevLett.112.144505 for an animation of the patch evolution in the rescaled time.

[19] D. G. Dritschel, P. H. Haynes, M. N. Juckes, and T. G. Shepherd, J. Fluid Mech. 230, 647 (1991). 